\title{
Inducción de micronúcleos y otras anormalidades nucleares en Astyanax gr. bimaculatus (Pisces: Characidae) expuestas a fenantreno
}

\author{
Induction of micronuclei and other nuclear abnormalities in \\ Astyanax gr. bimaculatus (Pisces: Characidae) \\ exposed to phenanthrene
}

\author{
Indução de micronúcleos e de outras anormalidades \\ nucleares em Astyanax gr. bimaculatus \\ (Pisces: Characidae) expostos a fenantreno
}

\author{
Wilson Corredor-Santamaría ${ }^{1}$, Cristián C. Mora-Romero²; Paula S. Escobar-Buitrago', \\ Pablo E. Cruz-Casallas ${ }^{3}$, Yohana M. Velasco-Santamaría ${ }^{4}$

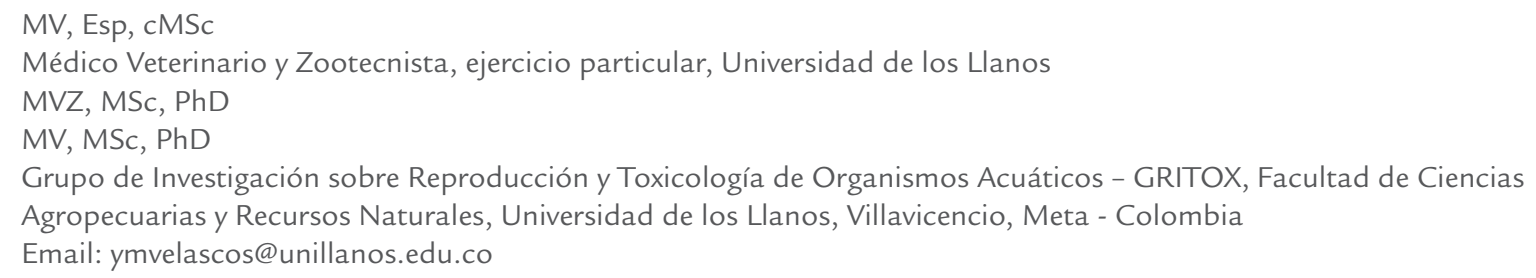

Recibido: septiembre 4 de 2012

Aceptado: noviembre 29 de 2012

\begin{abstract}
Resumen
El fenantreno es un hidrocarburo policíclico aromático (HPA) asociado a la actividad petrolera, altamente lipofílico, con amplia distribución y con la propiedad de persistir en el ambiente por tiempos prolongados. A pesar de su impacto, el efecto de los HPA ha sido poco estudiado en peces tropicales; por tanto, el objetivo de este estudio fue determinar la presencia de micronúcleos y otras anormalidades nucleares en eritrocitos de sangre periférica de Astyanax gr. bimaculatus expuestas a fenantreno. Los peces ( $\mathrm{n}=9$ ) fueron inyectados intraperitonealmente con 0.1, 1 y $10 \mu \mathrm{g} / \mathrm{g}$ de PV de fenantreno (PHE, Sigma Aldrich, pureza 98\%) disuelto en aceite vegetal, un control positivo inyectados con ciclofosfamida y un control negativo con aceite vegetal. Se colectaron muestras de sangre periférica a las 0 y 96 horas de la inyección. A las 96 horas, las dosis más altas de fenantreno mostraron efectos genotóxicos sobre los eritrocitos de Astyanax gr. bimaculatus, evidenciados por la formación de micronúcleos y alteraciones morfológicas nucleares. Estos resultados sugieren que Astyanax gr. bimaculatus, especie íctica dulceacuícola nativa, tiene gran potencial para ser utilizada como bioindicador de genotoxicidad causada por hidrocarburos policíclicos aromáticos.
\end{abstract}

Palabras clave: Aberración cromosómica, bioensayo, genotóxico, hidrocarburos aromáticos policíclicos, toxicidad. 


\begin{abstract}
Phenanthrene is a polycyclic aromatic hydrocarbon (PAH) associated with the oil industry, highly lipophilic, ubiquitous and with the property to persist in the environment for longer periods. Despite its impact, few studies has been carry out in tropical fish evaluating the PAH effect, therefore, the aim of this study was to determine the presence of micronucleus and other nuclear abnormalities in peripheral blood erythrocytes in Astyanax gr. bimaculatus exposed to phenantrene. Fish $(n=9)$ were injected intraperitoneally with $0.1,1$ and $10 \mu \mathrm{g} / \mathrm{g} \mathrm{BW}$ of phenantrene solution. Cicliphosphamide and vegetable oil were used as a positive and negative control, respectively. Peripheral blood samples were collected at 0 and 96 hours. At 96 h exposure, the two higher phenantrene concentrations showed genotoxic effect on Astyanax gr. bimaculatus erythrocytes caused by the micronucleus induction and other nuclear morphological alterations. These results suggest that Astyanax gr. bimaculatus, a native freshwater fish, has a great potential as a bioindicator of polycyclic aromatic hydrocarbons genotoxicity.
\end{abstract}

Key words: Chromosomal aberration, bioassays, genotoxic, polycyclic aromatic hydrocarbons, toxicity.

\title{
Resumo
}

Fenantreno é um hidrocarboneto aromático policíclico (HAP) associado com a indústria do petróleo, altamente lipofilico, com ampla distribuição e propriedade de persistirem no ambiente por longos períodos. No entanto seu impacto, o efeito dos HPA há sido pouco estudado em peixes tropicais, por tanto, o objetivo deste estudo foi determinar a presença dos micronúcleos e outras anormalidades nucleares em eritrócitos de sangue periférico do Astyanax gr. Bimaculatus expostas a fenantreno. Os peixes $(n=9)$ foram injetados intraperitonealmente com 0.1, 1 e $10 \mu \mathrm{g} / \mathrm{g}$ de fenantreno (PHE, Sigma Aldrich, 98\% de pureza), dissolvido em óleo vegetal, um controle positivo injetado com ciclofosfamida e um controlo negativo, injetado com o óleo vegetal. Amostras de sangue periférico foram coletadas as 0 e 96 horas de exposição. Às 96 horas, as doses mais altas de fenantreno mostraram efeitos genotóxicos sobre os eritrócitos de Astyanax gr. bimaculatus, evidenciados por a formação de micronúcleos e outras alterações morfológicas nucleares. Baseados nos resultados obtidos pode-se inferir que Astyanax gr. bimaculatus, peixe nativo de água doce, tem grande potencial para ser utilizado como um bioindicador de genotoxicidade, causada por hidrocarbonetos aromáticos policíclicos.

Palavras chave: Aberração cromossômica, hidrocarbonetos aromáticos policíclicos, genotóxicos, toxicidade.

\section{Introducción}

Los peces pueden actuar como organismos "centinela" para inferir el nivel de exposición de poblaciones humanas a sustancias químicas tóxicas presentes en los ecosistemas acuáticos (Kilgour et al., 2005). En este sentido, la realización de bioensayos para determinar los efectos de sustancias contaminantes de origen antropogénico o industrial, empleando peces como bioindicadores, proporciona información sobre los efectos de contaminantes químicos en ambientes acuáticos (Al-Sabti y Metcalfe, 1995; Matsumoto y Cólus, 2000), permitiendo un mejor monitoreo de las condiciones ambientales. Dentro de las especies más usadas como bioindicadores se encuentran pez cebra Danio rerio, carpa Cyprinus carpio, medaka Oryzias laticeps, trucha arcoíris Onchorrhynchus mykiss, sin embargo, estas especies foráneas solamente podrían usarse en estudios a nivel de laboratorio, limitando su aplicación en estudios de biomonitoreo en zonas tropicales. A este respecto, Astyanax bimaculatus, es una especie piscícola nativa de agua dulce no migratoria, pequeña, detritívora y con una amplia distribución en Sur América. Esta especie además de jugar un papel central en las redes alimentarias fluviales, es sensible a la degradación del medio ambiente, cuyas caracterís- ticas la convierten en un excelente bioindicador (Matsumoto y Cólus, 2000; Silva et al., 2010).

Dentro de las sustancias xenobióticas presentes en los ecosistemas acuáticos se encuentran los hidrocarburos aromáticos policíclicos (HAPs), los cuales son un grupo complejo de sustancias que tiene más de 10,000 compuestos individuales con 2 o más anillos de benceno fusionados (Boehm, 2005). La alta toxicidad de los HAPs se debe principalmente a los metabolitos tóxicos generados durante los procesos de biotransformación, los cuales establecen enlaces covalentes con macromoléculas de ADN, ARN y proteínas, generando efectos carcinogénicos y mutagénicos (Maioli et al., 2011; Tukivene, 1995).

El fenantreno (PHE) es un HAP con tres anillos, ampliamente distribuido en los ecosistemas terrestres y acuáticos por fuentes de origen antropogénico (Correia et al., 2007), que posee alta resistencia a la degradación química y biológica (Yuan et al., 2001). Debido a su toxicidad en un gran número de organismos acuáticos (Valavanidis et al., 2008; Yin et al., 2007), se ha considerado por la Environmental Protection Agency (USEPA) como uno de los 16 HAPs prioritarios para ser monitoreados (Anyakora et al., 2011). Debido a 
las características químicas del fenantreno, los peces pueden absorberlo del agua a través de las branquias y mediante la ingestión de sedimentos o alimentos contaminados (Reis-Henriques et al., 2009).

A pesar de la abundancia y ubicuidad del PHE en el medio acuático, la información es limitada sobre su toxicidad en peces (Oliveira et al., 2007). A este respecto, se han realizado estudios de exposición a PHE en peces teleósteos, usando la vía intraperitoneal (IP) con dosis variadas $(1,5,20 \mathrm{mg} / \mathrm{kg} \mathrm{PV})$ en especies como tilapia (Oreochromis niloticus), carpa común (Cyprinus carpio), bagre del canal (Ictalurus punctatus), fundulo (Fundulus heteroclitus) y tilapia negra (Oreochromis mossambicus) (Pathiratne y Hemachandra, 2010; Wahidulla y Rajamanickam, 2010; Whyte et al., 2000), utilizando aceite vegetal como vehículo (Shailaja et al., 2006).

El uso de inyecciones es frecuentemente usado para garantizar la exposición a sustancias químicas o tóxicas, siendo la inyección IP e IM los métodos más comunes en organismos acuáticos (Hylland et al., 1996; Kalman et al., 2010; Shailaja et al., 2006). La inyección intraperitoneal es una de las vías de administración más comunes para evaluar los efectos de sustancias altamente lipofílicas con alto potencial tóxico (CCAC, 2005; Hogan et al., 2010; Shailaja y D'Silva, 2003), particularmente en estudios de genotoxicidad en peces (Ayllon y Garcia-Vazquez, 2000).

Los micronúcleos (MN) son corpúsculos citoplasmáticos que contienen cromatina, los cuales pueden formarse durante la transición metafase/anafase de la mitosis al inhibirse el desplazamiento de los cromosomas en la anafase (evento aneugénico) o impedirse la fijación de las fibras del huso mitótico al cinetocoro, lo cual genera fragmentos cromosómicos acéntricos (evento clastogénico) que no se integran al núcleo de la célula hija después de la anafase (Miller et al., 1998). Estas alteraciones nucleares (AN) pueden ser inducidas mediante la exposición a sustancias tóxicas que impacten el ambiente natural (Ngan et al., 2007).

La presencia de micronúcleos es ampliamente utilizada como indicador de daño cromosómico y de inestabilidad del genoma, puesto que evidencia alteraciones en el huso cromático o en la estructura de los cromosomas; por lo tanto, desde hace aproximadamente 15 años, es una técnica usada con éxito en los estudios de biomonitoreo (larmarcovai et al., 2008).

Las alteraciones morfológicas nucleares ocurren por anormalidades durante la segregación de las cromátides en el proceso de división celular o durante la amplificación génica (Shimizu et al., 1998). Las anormalidades nucleares ( $\mathrm{MN}$, células binucleadas, núcleos lobed y núcleos blebbed) identificadas en los eritrocitos periféricos de los peces son consecuencia de agresiones genotóxicas o citotóxicas (Ayllon y Garcia-Vazquez, 2000; Çavaş y Ergene-Gözükara, 2003; Palhares y Grisolia, 2009; Souza y Fontanetti, 2006). Así mismo, el efecto de los HAPs ha sido estudiado In situ, donde se reporta mayor presencia de $\mathrm{MN}$ y AN en sangre periférica de peces que habitan ambientes susceptibles de contaminación por efluentes de refinerías petroleras (Souza y Fontanetti, 2006; Torres et al., 2008). A pesar que Colombia es un país con una alta explotación petrolera, son escasos los estudios orientados a determinar los efectos que compuestos derivados del petróleo ocasionan en organismos acuáticos. En este contexto, el objetivo del presente trabajo fue evaluar el efecto del fenantreno en dosis subletales como inductor de genotoxicidad, mediante el ensayo de micronúcleos y otras alteraciones nucleares en eritrocitos de sangre periférica del pez neotropical Astyanax gr. bimaculatus.

\section{Materiales y métodos}

\section{Material biológico}

Se utilizaron ejemplares de Astyanax gr. bimaculatus (Pisces: Characidae) de $8.5 \pm 0.4 \mathrm{~g}$ de peso corporal, procedentes de la estación piscícola del Instituto de Acuicultura (IALL) de la Universidad de los Llanos, Villavicencio, Colombia. El clima de la zona se caracteriza por una altura promedio de 418 m.s.n.m, con una temperatura máxima de $33,3{ }^{\circ} \mathrm{C}$ y una temperatura mínima promedio de $18,8{ }^{\circ} \mathrm{C}$, precipitación anual entre 1900 y 2300 mm y humedad relativa del 75\% (IDEAM, 2010). Previo a los experimentos, los peces fueron aclimatados a las condiciones de laboratorio durante un periodo de 15 días.

\section{Bioensayo}

El ensayo de toxicidad se llevó a cabo en el laboratorio de bioensayos del Grupo de investigación sobre Reproducción y Toxicología de Organismos Acuáticos - GRITOX de la Universidad de los Llanos. Los peces fueron alojados aleatoriamente en acuarios de vidrio (3 individuos por acuario) con capacidad de $20 \mathrm{~L} \mathrm{y}$ acondicionados con aireación constante. Se empleó un sistema semiestático, con recambio diario del $30 \%$ del volumen de agua, temperatura constante $\left(26^{\circ} \mathrm{C}\right)$ y fotoperiodo $12 \mathrm{~h}$ luz $/ 12 \mathrm{~h}$ oscuridad. Los peces fueron alimentados una vez al día con concentrado comercial a razón del 3\% de la biomasa. Diariamente, dos veces 
al día, fue monitoreado el oxígeno disuelto $(\mathrm{mg} / \mathrm{L})$ y la temperatura del agua $\left({ }^{\circ} \mathrm{C}\right)$, utilizando una sonda multiparamétrica YSI (MPS YSI 556). Además se midió el $\mathrm{pH}$ con un $\mathrm{pH}$-meter HANNA y la alcalinidad $(\mathrm{mg} / \mathrm{dL})$, dureza $(\mathrm{mg} / \mathrm{dL})$ y amonio $(\mathrm{mg} / \mathrm{L})$, usando un kit colorimétrico (HACH Fish Farming Model FF-1A.).

\section{Diseño Experimental}

Los peces $(n=9)$ fueron inyectados intraperitonealmente con tres dosis subletales $(0.1,1$ y $10 \mu \mathrm{g} / \mathrm{g}$ PV) de fenantreno (PHE, Sigma Aldrich ${ }^{\circledR}$, pureza 98\%) disuelto en aceite vegetal. Estas dosis fueron seleccionadas de acuerdo a estudios previos con fenantreno los cuales reportan en otras especies de peces el uso de dosis

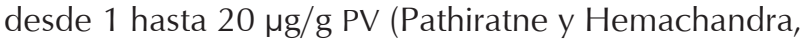
2010; Wahidulla y Rajamanickam, 2010; Whyte et al., 2000).

El experimento tuvo una duración de 96 horas. Adicional a las 3 dosis de PHE, se establecieron dos grupos control, un control positivo con ciclofosfamida (Endoxan Baxter $^{\circledR}, n=9$ ), agente alquilante inhibidor de la síntesis de ADN, a una dosis de 20 mg/g PV (Grisolia, 2002) inyectado IP con solución salina al $0.9 \%$ y un control negativo que consistió en administrar solamente el vehículo (aceite vegetal, $n=9$ ). Se manejaron tres replicas por tratamiento, siendo la unidad experimental el pez, para un total de 45 peces.

A las 0 y 96 horas de la inyección, los peces fueron anestesiados con 2-fenoxietanol (300 ppm, JT Baker ${ }^{\circledR}$ ) para obtener muestras de sangre mediante punción de los vasos sanguíneos caudales con jeringas heparinizadas. Transcurridas las 96 h desde la inyección, los peces fueron anestesiados, la sangre colectada y posteriormente sacrificados para realizar necropsia detallada, con especial énfasis en lesiones macroscópicas de hígado y branquias.

\section{Anormalidades nucleares}

\section{Prueba de micronúcleos}

A partir de las muestras de sangre se realizaron dos frotis sanguíneos por animal para determinar la frecuencia de $M N$ y de alteraciones nucleares. Los extendidos sanguíneos fueron teñidos durante 10 min con colorante de Wright-Metanol $\left(\right.$ Merck $\left.^{\circledR}\right)$ previamente filtrado (Papel de filtro $3 \mu \mathrm{m}$, Whatman ${ }^{\circledR}$ ).

La lectura de las láminas se efectuó a ciegas, contando 2,000 células por frotis. Sólo se tuvieron en cuenta células sanguíneas con membranas nucleares y citoplas- máticas intactas, descartando aquellas sobrepuestas o dañadas (Al-Sabti y Metcalfe, 1995). Para identificar los MN se tomaron los siguientes criterios: diámetro del MN menor a un $1 / 3$ del núcleo principal, estar claramente separado del núcleo, no ser refractario, presentar su mismo color e intensidad y estar incluidos en el citoplasma celular (Grisolia, 2002).

El conteo de MN fue expresado en porcentaje (MNC $\%)$, calculado a partir del número de eritrocitos micronucleados observados por cada 2000 células.

$$
\text { MNC\% }=\frac{N^{o} \text { de células con MN }}{2000} \times 100
$$

Así mismo, se calculó el índice de proliferación (IP) que se obtiene considerando el \% de MNC del control negativo respecto al \% de MNC calculado en las muestras experimentales.

$$
I \mathrm{P} \%=\frac{\text { MNC } \% \text { de las muestras }}{\text { MNC } \% \text { del control negativo }}
$$

\section{Otras alteraciones morfológicas nucleares}

Las alteraciones morfológicas nucleares en eritrocitos fueron clasificadas de acuerdo con los criterios de Carrasco et al. (1990), así: núcleos que presentan una pequeña invaginación en la membrana nuclear y contienen eucromatina llamados blebbed (BL), células binucleadas (BN), núcleos con características de micronúcleo conectados al núcleo por un tallo de material nucleoplásmico denominados lobed (LB). La observación de MN y AN se realizó bajo un microscopio óptico (Nikon eclipse E-400) con magnificación de 1000X.

\section{Diámetro eritrocitario}

La determinación del tamaño eritrocitario se efectuó capturando imágenes de cada extendido sanguíneo con una cámara fotográfica digital Nikon, acoplada a un microscopio (Nikon eclipse E-400). El diámetro eritrocitario se calculó con el software Image I (National Institutes of Health, USA), previamente calibrado, midiendo 100 eritrocitos por frotis.

\section{Análisis estadístico}

Los resultados son mostrados como media \pm error estándar de la media (SEM). La normalidad y homogeneidad de varianza se evaluó por medio del test de Kolmogorov-Smirnov y Bartlett's, respectivamente. En cada individuo se determinó la frecuencia de micronúcleos y de otras anormalidades nucleares. Al no cumplirse los supuestos de normalidad y de homogeneidad 
de varianza, los datos fueron transformados a ranks y posteriormente sometidos a un ANOVA de dos vías para evaluar los factores de manera individual y sus interacciones, donde el factor 1 correspondió a los tratamientos inyectados (control negativo, control positivo y $0,1,1$ y $10 \mu \mathrm{g} / \mathrm{g}$ PV PHE) y el factor 2 correspondió al tiempo de exposición (0 y 96 h). Finalmente se realizó la prueba de Tukey para comparar las diferencias entre los factores. Un valor de $\mathrm{P}<0.05$ fue considerado significativo. Para el análisis estadístico fue empleado el software SAS versión 9.2 para Windows (2002-2008 por SAS Institute Inc., Cary, NC, USA).

\section{Resultados}

Después de 96 h de administrados los tratamientos, no se observó mortalidad ni lesiones macroscópicas durante la necropsia en ninguno de los peces expuestos a las dosis subletales de fenantreno, ni en aquellos expuestos a la ciclofosfamida o utilizados como grupo control negativo.

\section{Parámetros fisicoquímicos}

Los parámetros de calidad de agua no presentaron diferencias significativas en ninguno de los tratamientos durante las $96 \mathrm{~h}$ del ensayo. La temperatura del agua fluctuó entre 25 y $28{ }^{\circ} \mathrm{C}$, el pH entre 7.2 y 7.5 , la dureza entre 28.5 y $29.6 \mathrm{mg} / \mathrm{L}$, la alcalinidad entre 25.1 y $31.9 \mathrm{mg} / \mathrm{L}$ y el amonio entre 0.4 y $0.6 \mathrm{mg} / \mathrm{L}$.

\section{Variables de genotoxicidad}

\section{Prueba de micronúcleos}

En la figura 1 se observa el porcentaje de $M N$ en eritrocitos de Astyanax gr. bimaculatus expuestos a diferentes dosis de fenantreno. El ANOVA de dos vías reveló diferencias significativas entre los tratamientos, entre las horas de exposición y su respectiva interacción. A la hora 0 , el mayor recuento de MN se observó en los peces expuestos a $1 \mathrm{\mu g} / \mathrm{g}$ de PHE, aunque en general los porcentajes de $\mathrm{MN}$ estuvieron por debajo de $0.5 \%$. Después de las $96 \mathrm{~h}$ fue evidente el incremento en el porcentaje de $\mathrm{MN}$, con diferencias extremadamente significativas $(\mathrm{P}<0.001)$ entre los grupos experimentales cuando comparado con la hora 0 de exposición. Todos los peces expuestos a PHE presentaron los mayores recuentos de micronúcleos, sin diferencias significativas con el grupo expuesto a ciclofosfamida $(\mathrm{P}>0.05)$. Los peces del grupo control negativo presentaron la menor frecuencia de $\mathrm{MN}$ a las 96 horas de exposición, siendo diferente estadísticamente con los demás tratamientos $(\mathrm{P}<0.05)$ a excepción del grupo tratado con 1 $\mu \mathrm{g} / \mathrm{g}$ PV de PHE (P>0.05).

El índice PI obtenido a partir de la comparación entre el índice $\mathrm{MNC} \%$ y su respectivo control, arrojó una mayor variación entre los grupos expuestos a 10 y 0.1 $\mu \mathrm{g} / \mathrm{g}$ PV de PHE frente al control negativo, hallando un

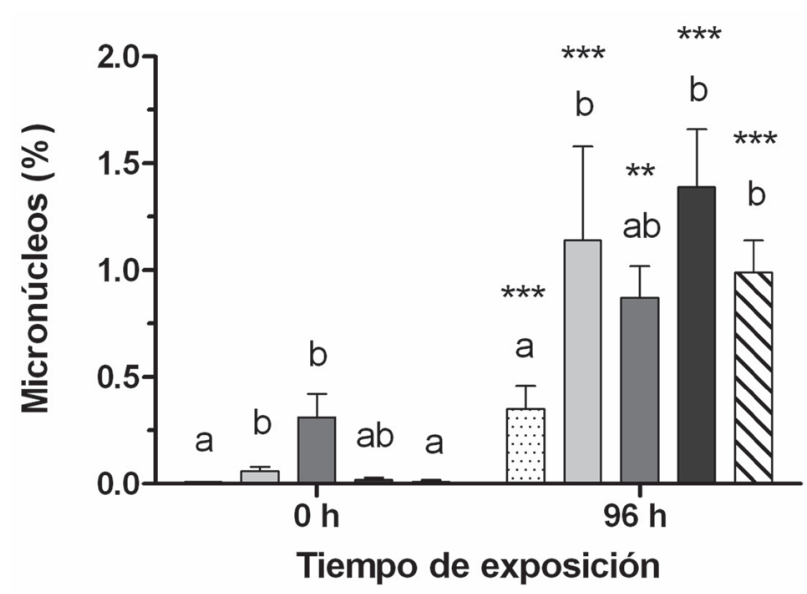

\section{Control negativo $\quad \Delta$ Control positivo \\ $0.1 \mu \mathrm{g} / \mathrm{g} \square 1 \mu \mathrm{g} / \mathrm{g} \square 10 \mu \mathrm{g} / \mathrm{g}$}

Figura 1. Frecuencia de $M N$ en eritrocitos de sangre periférica de Astyanax gr. bimaculatus después de 96 h de la exposición a $0.1,1$ y $10 \mu \mathrm{g} / \mathrm{g}$ PV de fenantreno y en dos controles, control positivo: ciclofosfamida $(0.20 \mu \mathrm{g} / \mathrm{g}$ PV) y control negativo: aceite vegetal. $(n=9)$. ${ }^{a, b}$ Para la misma hora de muestreo, letras diferentes entre barras indican diferencias significativas $(P<0.05)$. ${ }^{*}$ Indica diferencias significativas entre las 0 y 96 horas, * $(\mathrm{P}<0.05) ; * *(\mathrm{P}<0.01) ; * * *(\mathrm{P}<0.001)$. 
PI entre 3.24 y 3.97 , el cual aumentó a medida que se incrementó la concentración de fenantreno.

\section{Otras alteraciones morfológicas nucleares}

$\mathrm{Al}$ igual que los $\mathrm{MN}$, las demás alteraciones nucleares presentaron diferencias significativas entre los tratamientos, entre las horas de exposición y su respectiva interacción (ANOVA de dos vías, $\mathrm{P}<0.05$ ).

\section{Núcleos lobulados (Lobed)}

Al inicio del ensayo no se observaron diferencias significativas entre tratamientos con respecto a la frecuencia de núcleos lobulados. A las 96 horas de exposición se observó un evidente efecto dosis-respuesta a medida que aumentó la concentración de PHE, siendo los peces expuestos a $10 \mathrm{\mu g} / \mathrm{g}$ de PHE los que presentaron el mayor porcentaje de núcleos lobed, similar al porcentaje observado en el control positivo con ciclosfofamida $(\mathrm{P}<0.05)$. Solo los peces expuestos a ciclofosfamida presentaron diferencias significativas entre las 0 y $96 \mathrm{~h}$ de exposición ( $\mathrm{P}<0.01$, Fig. $2 \mathrm{~A})$.

\section{Núcleos con hendidura (Blebbed)}

En cuanto a las anormalidades nucleares con núcleo en forma de hendidura, no se hallaron diferencias significativas al inicio (hora 0 ) del ensayo. No obstante, a las
$96 \mathrm{~h}$ se presentaron diferencias significativas $(\mathrm{P}<0.05)$ entre los peces sometidos a la dosis de $10 \mu \mathrm{g} / \mathrm{g}$ PV de PHE cuando comparadas con la menor concentración. Se observó una tendencia monotónica en los peces expuestos a PHE posterior a las $96 \mathrm{~h}$. Entre tiempos de exposición, para esta anormalidad nuclear, solo se encontraron diferencias significativas en el grupo expuesto a $10 \mu \mathrm{g} / \mathrm{g}$ de $\mathrm{PHE}$ ( $\mathrm{P}<0.05$, Fig. 2B).

\section{Células binucleadas}

Con referencia a la frecuencia de eritrocitos con células binucleadas, se observó a las 0 horas un patrón similar a las variables de eritrocitos con núcleo tipo lobed y blebbed. Del mismo modo, no se observaron diferencias significativas entre ninguno de los tratamientos a las $96 \mathrm{~h}$ de exposición, aunque la mayor frecuencia de células binucleadas fue observada a la mayor concentración de fenantreno (Fig. 3A). Entre tiempos de exposición, todos los tratamientos presentaron diferencias significativas $(\mathrm{P}<0.05)$.

El consolidado de anormalidades nucleares (núcleos lobed, núcleos blebbed y células binucleadas) se puede apreciar en la figura 3B. Al inicio del ensayo no se hallaron diferencias significativas entre ninguno de los grupos expuestos $(\mathrm{P}<0.05)$. Al finalizar el ensayo, se observó un claro efecto dosis-respuesta de los grupos expuestos a PHE con diferencias significativas $(\mathrm{P}<0.05)$. Pero únicamente los peces expuestos a $10 \mu \mathrm{g} / \mathrm{g}$ PV
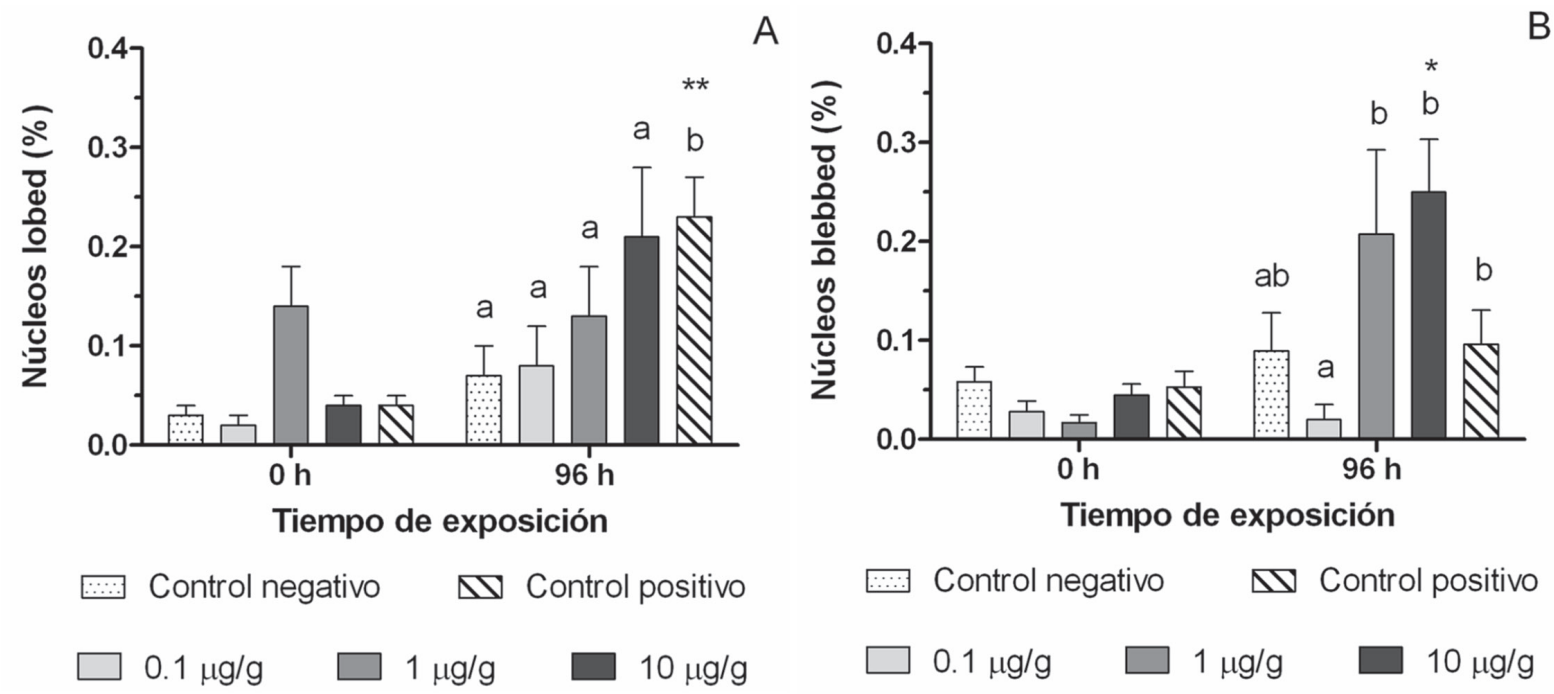

Figura 2. Frecuencia de eritrocitos con núcleos lobed (LB) (A) y con núcleos blebbed (B) de Astyanax gr. bimaculatus, después de 96 de la exposición a 0.1, 1 y $10 \mu \mathrm{g} / \mathrm{g}$ PV de fenantreno y a dos controles, control positivo: ciclofosfamida $(0.20 \mu \mathrm{g} / \mathrm{g}$ PV) y control negativo: aceite vegetal. $(n=9)$. a,b Para la misma hora, letras diferentes entre barras indican diferencias significativas $(P<0.05)$. * Indica diferencias significativas entre las 0 y 96 horas, ${ }^{*}(P<0.05) ;{ }^{* *}(P<0.01) ;{ }^{* * *}(P<0.001)$. 
de PHE presentaron diferencias con respecto al grupo control negativo $(P<0.05)$. Las mayores anormalidades nucleares fueron observadas en los peces expuestos a 1 y $10 \mu \mathrm{g} / \mathrm{g}$ PV de PHE y ciclofosfamida, sin encontrarse diferencias significativas entre estos grupos. Al comparar las 0 y $96 \mathrm{~h}$, solo se observaron diferencias significativas en los peces expuestos a $10 \mathrm{\mu g} / \mathrm{g}$ PV de PHE y los expuestos a ciclofosfamida ( $\mathrm{P}<0.01$, Fig. 3B).

\section{Determinación del diámetro eritrocitario}

En cuanto al diámetro eritrocitario de Astyanax gr. bimaculatus, al inicio del ensayo se observó que los peces del grupo control negativo y expuestos a $0.1 \mathrm{\mu g} / \mathrm{g}$ PV de PHE presentaron los mayores diámetros frente a las demás grupos experimentales $(\mathrm{P}<0.05)$. A las 96 h, los mayores diámetros celulares fueron encontrados en el grupo expuesto a $1 \mu \mathrm{g} / \mathrm{g}$ PV de PHE y en el control expuesto a ciclofosfamida, diferentes significativamente cuando comparado con el grupo control negativo y $10 \mu \mathrm{g} / \mathrm{g}$ PV de PHE $(\mathrm{P}<0.05)$. Igualmente, fueron encontradas diferencias altamente significativas $(\mathrm{P}<0.001)$ entre los dos tiempos de exposición (0 y 96 h) para todos los grupos expuestos, excepto los peces expuestos a $0.1 \mu \mathrm{g} / \mathrm{g}$ PV de PHE (Fig. 4).

\section{Discusión}

En este estudio se observaron efectos genotóxicos ocasionados por el fenantreno y la ciclofosfamida a través de la inyección IP con dosis subletales, los cua- les indujeron la formación de un número considerable de micronúcleos y de anormalidades nucleares en eritrocitos de sangre periférica de ejemplares de Astyanax gr. bimaculatus, corroborando la eficacia de la ciclofosfamida como control positivo en estudios de genotoxicidad.

Aunque la inyección no es la ruta natural de exposición, el uso de inyecciones principalmente intraperitoneal es una de las más usadas en ecotoxicología ya que garantiza una liberación directa de la sustancia a evaluar, reduce los posibles efectos externos ocasionados por condiciones fisicoquímicas y minimiza los riesgos de contaminación ambiental (Karami et al., 2011). Entre los efectos no controlables de la inmersión pueden citarse las pérdidas por volatilización, absorción y metabolismo (Prosser et al., 2011). A este respecto, en O. mossambicus se evaluó la respuesta a la exposición a PHE usando la vía de administración IP (1 Mg/g PV) e inmersión (1 mg/L); aunque ambas exposiciones indujeron la formación de ciertos tipos de aductos de ADN (etapas primarias de procesos mutagénicos) en bilis, la inyección IP indujo los mayores efectos (Wahidulla y Rajamanickam, 2010). En peces no se reporta $\mathrm{CL}_{50}$ de PHE vía IP; sin embargo, la Agencia de Protección Ambiental de Canadá (CEPA, 1994), reportó en juveniles de trucha arcoíris una $\mathrm{CL}_{50}$ a 96 horas de $375 \mu \mathrm{g} / \mathrm{L}$.

El ensayo de $M N$ en eritrocitos periféricos de diferentes especies de peces como biomarcador de los

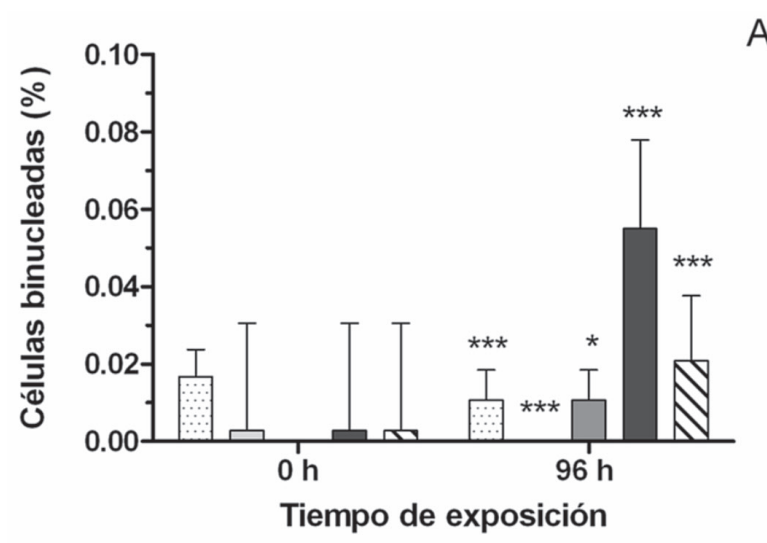

$$
\begin{aligned}
& \text { Control negativo } \quad \square \text { Control positivo } \\
& 0.1 \mu \mathrm{g} / \mathrm{g} \quad \square 1 \mu \mathrm{g} / \mathrm{g} \quad \square 10 \mu \mathrm{g} / \mathrm{g}
\end{aligned}
$$

A

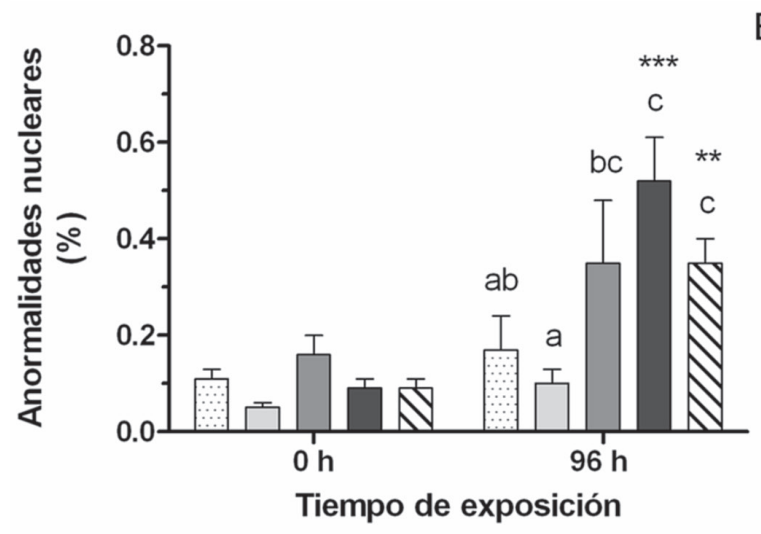

Control negativo

$\Delta$ Control positivo

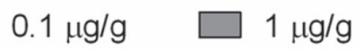

B

Figura 3. Frecuencia de células binucleadas (A) y anormalidades nucleares (B) de Astyanax gr. bimaculatus, después de la exposición a $0.1,1$ y $10 \mu \mathrm{g} / \mathrm{g}$ PV de fenantreno y a dos controles, control positivo: ciclofosfamida $(0.20 \mu \mathrm{g} / \mathrm{g}$ PV) y control negativo: aceite vegetal. $(n=9) .{ }^{a, b, c}$ Para la misma hora, letras diferentes entre barras indican diferencias significativas $(P<0.05) .{ }^{*}$ Indica diferencias significativas entre las 0 y 96 horas, ${ }^{*}(\mathrm{P}<0.05) ;{ }^{* *}(\mathrm{P}<0.01) ;{ }^{* * *}(\mathrm{P}<0.001)$. 


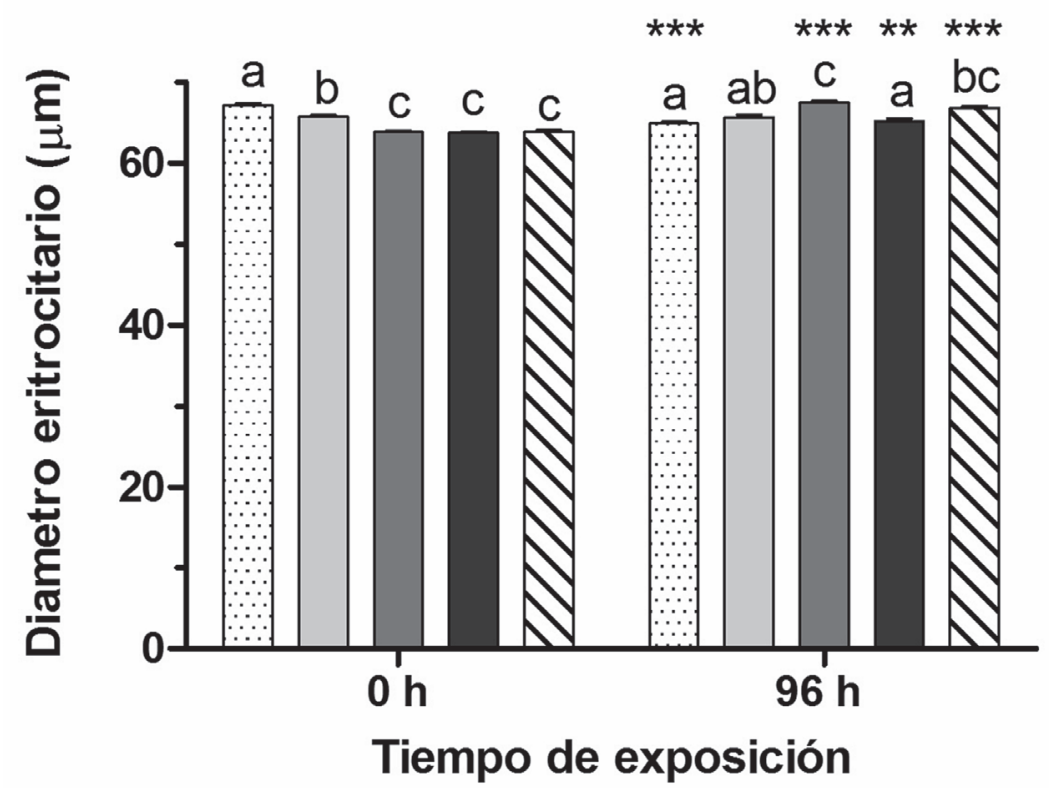

.... Control negativo $\Delta \nabla$ Control positivo

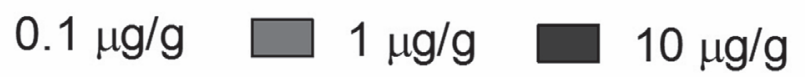

Figura 4. Diámetro eritrocitario en Astyanax gr. bimaculatus, después de la exposición a $0.1,1$ y $10 \mu \mathrm{g} / \mathrm{g}$ PV de fenantreno y a dos controles, control positivo: ciclofosfamida $(0.20 \mu \mathrm{g} / \mathrm{g}$ PV) y control negativo: aceite vegetal. ( $\mathrm{n}=9)$. ${ }^{\mathrm{a}, \mathrm{b}, \mathrm{c}}$ Para la misma hora, letras diferentes entre barras indican diferencias significativas $(P<0.05) .{ }^{*}$ Indica diferencias significativas entre las 0 y 96 horas, ${ }^{*}(\mathrm{P}<0.05) ;{ }^{* *}(\mathrm{P}<0.01) ;{ }^{* * *}(\mathrm{P}<0.001)$.

efectos mutagénicos de los HAPs se ha evaluado bajo condiciones controladas de laboratorio registrando la presencia de $\mathrm{MN}$ y AN, observándose una relación dosis-respuesta (Baršienè et al., 2006; Hoshina et al., 2008; Vanzella et al., 2007). Las alteraciones morfológicas nucleares encontradas en este estudio fueron consideradas biomarcadores de genotoxicidad (Ayllon y García-Vázquez, 2000; Çavaş y Ergene-Gözükara, 2003; Palhares y Grisolia, 2009). Se ha demostrado la inducción de $\mathrm{MN}$ y AN en eritrocitos de especies ícticas como lenguado Solea senegalensis y tilapia $O$. niloticus, exponiéndolos a efluentes de refinerías petroleras, los cuales están constituidos en una importante proporción por hidrocarburos aromáticos policíclicos HAPs (Costa et al., 2011; Souza y Fontanetti, 2006).

Reis-Henriques et al. (2009) reportaron la formación de MN en róbalos Dicentrarchus labrax expuestos a una concentración de $10 \mathrm{\mu g} / \mathrm{g}$ de PHE. Por otro lado, Brzuzan et al. (2006) en Oncorhynchus mykiss evaluaron vía IP $10 \mu \mathrm{g} / \mathrm{g}$ de ciclopenta-fenantreno y hallaron una correlación positiva entre la frecuencia de anormalidades nucleares blebbed y los niveles de expresión de
RNAm de CYP1a, demostrando la inducción del PHE en los procesos de biotransformación I en peces teleósteos. Resultados similares fueron observados en el presente estudio, en el cual la concentración de 1 y 10 $\mu \mathrm{g} / \mathrm{g}$ de PHE indujeron la presencia de anormalidades nucleares en Astyanax gr. bimaculatus. A nivel nacional no existen reportes de la inducción de MN por exposición a PHE en especies ícticas dulceacuícolas, siendo este estudio uno de los pioneros a este respecto.

Puede considerarse que los MN observados en todos los tratamientos a las 0 horas y en los peces del control negativo a las $96 \mathrm{~h}$, pueden estar asociados a una inducción espontánea, ya que estos peces no fueron expuestos previamente a sustancias genotóxicas. Aunque la presencia espontánea de micronúcleos en peces es rara, se han reportado estudios con tasas de eritrocitos con $\mathrm{MN}$ espontáneos entre 6.2 y 13,7 por 1000 eritrocitos de Rhamdia quelen, Cyprinus carpio, Hyposthomus punctatus y Oreochromis niloticus (Salvagni et al., 2011), hasta $3.7 \mathrm{MN}$ en 1000 eritrocitos de trucha café (Salmo trutta), anguila europea (Anguilla anguilla) y foxino (Phoxinus phoxinus) (Rodriguez-Cea 
et al., 2003) y de $3 \mathrm{MN}$ en 2000 eritrocitos de Astyanax bimaculatus (Matsumoto y Cólus, 2000).

Oliveira et al. (2007) reportaron la inducción de AN en Liza aurata durante cortos periodos de exposición $(16 \mathrm{~h})$ y en concentraciones subletales $(0.1,0.3,0.9$ y $2.7 \mu \mathrm{M})$ de PHE; estos autores observaron un aumento progresivo en la presentación de anormalidades asociadas al incremento de las concentraciones del tóxico. Del mismo modo, en el presente estudio se observó un claro efecto dosis-respuesta en la formación de anormalidades en eritrocitos periféricos de Astyanax gr. bimaculatus, a medida que se incrementó la concentración de PHE (Fig. 3B).

En cuanto a la presencia de núcleos blebbed y el consolidado de AN en los eritrocitos de Astyanax gr. bimaculatus expuestos a PHE y su relación monotónica dosis-respuesta con PHE, se puede deducir que dichas anormalidades pueden estar asociadas con un efecto clastogénico del PHE (Brzuzan et al., 2006). La presencia de células binucleadas es considerada indicador de citotoxicidad (Çavaş y Ergene-Gözükara, 2005), las cuales aparecen debido a un bloqueo de la citoquinesis durante la división celular anormal, generando un desbalance genético en las células, el cual puede predisponer a carcinogénesis (Rodilla, 1993); aunque en el presente estudio las diferencias no fueron significativas, se observó un mayor número de células binucleadas en la sangre de Astyanax gr. bimaculatus expuestos a $10 \mu \mathrm{g} / \mathrm{g}$ de PHE, lo que podría indicar un posible efecto citotóxico del PHE.

El aumento significativo en el diámetro eritrocitario en los peces expuestos a las dosis de $1 \mu \mathrm{g} / \mathrm{g}$ de PHE, podría estar relacionado con una reacción compensatoria en cortos periodos de tiempo de los órganos hematopoyéticos que provoca procesos progresivos de hipertrofia en los eritrocitos (Arnaudov et al., 2008).

En conclusión, el fenantreno en dosis subletales es un agente inductor de genotoxicidad y citotoxicidad sobre los eritrocitos de sangre periférica de Astyanax gr. bimaculatus. Estos resultados sugieren que la presencia de este hidrocarburo aromático policíclico en bajas dosis, puede desencadenar aberraciones cromosómicas y daños citotóxicos en peces que se encuentren expuestos a estas concentraciones.

\section{Agradecimientos}

Los autores agradecen a todas las personas que apoyaron el desarrollo experimental del presente estudio. Así como el agradecimiento al Instituto de Acuicultura de la Universidad de los Llanos por el apoyo logísti- co para la realización de este estudio. Este estudio se realizó en el marco del Convenio No. 008 de 2008, suscrito entre ECOPETROL-ICP - UNILLANOS, para la ejecución del proyecto de investigación titulado: "Evaluación de una especie fitoplanctónica nativa de la Orinoquia colombiana como bioindicador de la toxicidad en los cuerpos de aguas de uso potencial para la acuicultura.

\section{Referencias}

Al-Sabti K, Metcalfe CD. Fish micronuclei for assessing genotoxicity in water. Mutation Research. 1995; 343: 121-135.

Anyakora C, Coker H, Arbabi M. Application of polynuclear aromatic hydrocarbons in chemical fingerprinting: the niger delta case study. Iranian Journal of Environmental Health Science y Engineering. 2011; 8: 75-84.

Arnaudov AD, Velcheva IG, Tomova ES. Influence of copper and zinc on the erythrocyte metric parameters of carassius gibelio (pisces, cyprinidae) Bulgarian Journal of Agricultural Science. 2008; 14: 557-563.

Ayllon F, Garcia-Vazquez E. Induction of micronuclei and other nuclear abnormalities in European minnow Phoxinus phoxinus and mollie Poecilia latipinna: an assessment of the fish micronucleus test. Mutation Research/Genetic Toxicology and Environmental Mutagenesis. 2000; 467: 177-186.

Baršienñé J, Dedonyté V, Rybakovas A, Andreikénaité L, Andersen OK. 2006. Investigation of micronuclei and other nuclear abnormalities in peripheral blood and kidney of marine fish treated with crude oil. Aquatic Toxicology. 78, Supplement: S99-S104.

Brzuzan P, Jurczyk L, Wozny M. Molecular geometry, CYP1A gene induction and clastogenic activity of cyclopenta [C] phenanthrene in rainbow trout. Polycyclic Aromatic Compounds. 2006; 26: 345-365.

Carrasco KR, Tilbury KL, Myers MS. Assessment of the Piscine Micronucleus Test as an in situ Biological indicator of Chemical Contaminant Effects. Canadian Journal of Fisheries and Aquatic Sciences. 1990; 47: 2123-2136.

Çavaş T, Ergene-Gözükara S. Micronuclei, nuclear lesions and interphase silver-stained nucleolar organizer regions (AgNORs) as cyto-genotoxicity indicators in Oreochromis niloticus exposed to textile mill effluent. Mutation Research/Genetic Toxicology and Environmental Mutagenesis. 2003; 538: 81-91.

Çavaş T, Ergene-Gözükara S. Induction of micronuclei and nuclear abnormalities in Oreochromis niloticus following exposure to petroleum refinery and chromium processing plant effluents. Aquatic Toxicology. 2005; 74: 264-271.

Correia AD, Gonçalves R, Scholze M, Ferreira M, Henriques MAR. Biochemical and behavioral responses in gilthead seabream (Sparus aurata) to phenanthrene. Journal of Experimental Marine Biology and Ecology. 2007; 347: 109-122.

Costa PM, Neuparth TS, Caeiro S, Lobo J, Martins M, Ferreira AM, Caetano M, Vale C, Ángel DelValls T, Costa MH. Assessment 
of the genotoxic potential of contaminated estuarine sediments in fish peripheral blood: Laboratory versus in situ studies. Environmental Research. 2011; 111: 25-36.

Grisolia CK. A comparison between mouse and fish micronucleus test using cyclophosphamide, mitomycin $\mathrm{C}$ and various pesticides. Mutation Research/Genetic Toxicology and Environmental Mutagenesis. 2002; 518: 145-150.

Hogan NS, Lee KS, Köllner B, van den Heuvel MR. The effects of the alkyl polycyclic aromatic hydrocarbon retene on rainbow trout (Oncorhynchus mykiss) immune response. Aquatic Toxicology. 2010; 100: 246-254.

Hoshina MM, de Angelis DdF, Marin-Morales MA. Induction of micronucleus and nuclear alterations in fish (Oreochromis niloticus) by a petroleum refinery effluent. Mutation Research/ Genetic Toxicology and Environmental Mutagenesis. 2008; 656: 44-48

Hylland K, Sandvik M, Skare JU, Beyer J, Egaas E, Goksoyr A. Biomarkers in flounder (Platichthys $\square$ esus): an evaluation of their use in pollution monitoring. Marine Environment Research. 1996; 42: 223-227.

larmarcovai G, Bonassi S, Botta A, Baan RA, Orsière T. Genetic polymorphisms and micronucleus formation: $\mathrm{A}$ review of the literature. Mutation Research/Reviews in Mutation Research. 2008; 658: 215-233.

Kalman J, Riba I, Ángel DelValls T, Blasco J. Comparative toxicity of cadmium in the commercial fish species Sparus aurata and Solea senegalensis. Ecotoxicology and Environmental Safety. 2010; 73: 306-311.

Karami A, Christianus A, Ishak Z, Syed MA, Courtenay SC. The effects of intramuscular and intraperitoneal injections of benzo[a]pyrene on selected biomarkers in Clarias gariepinus. Ecotoxicology and Environmental Safety. 2011; 74: 1558-1566.

Kilgour BW, Munkittrick KR, Culp J, Dixit S, Portt CB, Pastershank G. Biological Criteria for Municipal Wastewater Effluent Monitoring Programs. Water Quality Research Journal of Canada. 2005; 40: 374-387.

Maioli O, Rodrigues K, Knoppers B, Azevedo D. Distribution and sources of aliphatic and polycyclic aromatic hydrocarbons in suspended particulate matter in water from two Brazilian estuarine systems. Continental Shelf Research. 2011; 31: 11161127.

Matsumoto FE, Cólus IMS. Micronucleus frequencies in Astyanax bimaculatus (Characidae) treated with cyclophosphamide or vinblastine sulfate. Genetics and Molecular Biology. 2000; 23 489-492.

Miller B, Pötter-Locher F, Seelbach A, Stopper H, Utesch D, Madle $\mathrm{S}$. Evaluation of the in vitro micronucleus test as an alternative to the in vitro chromosomal aberration assay: position of the GUM working group on the in vitro micronucleus test. $\mathrm{Mu}$ tation Research/Reviews in Mutation Research. 1998; 410: 81-116.

Ngan P, Gomes V, Passos MCR, Ussami K, Campos DF, Rocha A, Pereira B. Biomonitoring of the genotoxic potential (micronucleus and erythrocyte nuclear abnormalities assay) of the Admiralty Bay water surrounding the Brazilian Antarctic Research
Station "Comandante Ferraz," King George Island. Polar Biology. 2007; 30: 209-217.

Oliveira M, Pacheco M, Santos MA. Cytochrome P4501A, genotoxic and stress responses in golden grey mullet (Liza aurata) following short-term exposure to phenanthrene. Chemosphere. 2007; 66: 1284-1291.

Palhares D, Grisolia CK. Comparison between the micronucleus frequencies of kidney and gill erythrocytes in tilapia fish, following mitomycin C treatment. Genetics and Molecular Biology. 2009; 25: 281-284.

Pathiratne A, Hemachandra CK. Modulation of ethoxyresorufin O-deethylase and glutathione S-transferase activities in Nile tilapia (Oreochromis niloticus) by polycyclic aromatic hydrocarbons containing two to four rings: implications in biomonitoring aquatic pollution. Ecotoxicology. 2010; 19: 1012-1018.

Prosser CM, Unger MA, Vogelbein WK. Multistressor interactions in the zebrafish (Danio rerio): Concurrent phenanthrene exposure and Mycobacterium marinum infection. Aquatic Toxicology. 2011; 102: 177-185.

Reis-Henriques MA, Ferreira M, Coimbra A, Silva CD, Costa J, Shailaja MS. Phenanthrene and nitrite effects on juvenile sea bass, Dicentrarchus labrax, using hepatic biotransformation enzymes, biliary fluorescence, and micronuclei as biomarkers. Ciencias Marinas. 2009; 35: 29-40.

Rodilla V. Origin and evolution of binucleated cells and binucleated cells with micronuclei in cisplatin-treated $\mathrm{CHO}$ cultures. Mutation Research/Genetic Toxicology. 1993; 300: 281-291.

Rodriguez-Cea A, Ayllon F, Garcia-Vazquez E. Micronucleus test in freshwater fish species: an evaluation of its sensitivity for application in field surveys. Ecotoxicology and Environmental Safety. 2003; 56: 442-448.

Salvagni J, Zeni R, Fuentefria A. Assesment of the genotoxic impact of persticides on farming communities in the countryside of Santa Catarina State, Brazil. Genetics and Molecular Biology. 2011; 34: 122-126.

Shailaja MS, D'Silva C. Evaluation of impact of PAH on a tropical fish, Oreochromis mossambicus using multiple biomarkers. Chemosphere. 2003; 53: 835-841.

Shailaja MS, Rajamanickam R, Wahidulla S. Formation of Genotoxic Nitro-PAH Compounds in Fish Exposed to Ambient Nitrite and PAH. Toxicological Sciences. 2006; 91: 440-447.

Shimizu N, Itoh N, Utiyama H, Vahl GM. Selective entrapment of extrachromosomally DNA by nuclear budding and micronucleation during the S phase. Cellular Biology. 1998; 140: 13071320.

Silva RRPd, Pires Junior OR, Grisolia CK. Toxicity and genotoxicity in Astyanax bimaculatus (Characidae) induced by microcystins from a bloom of Microcystis spp. Genetics and Molecular Biology. 2010; 33: 750-755.

Souza TDS, Fontanetti CS. Micronucleus test and observation of nuclear alterations in erythrocytes of Nile tilapia exposed to waters affected by refinery effluent. Mutation Research/Ge- 
netic Toxicology and Environmental Mutagenesis. 2006; 605: 87-93.

De Lemos CT, Iranço FdeA, de Oliveira NC, de Souza GD, Fachel JMG. Biomonitoring of genotoxicity using micronuclei assay in native population of Astyanax jacuhiensis (Characiformes: Characidae) at sites under petrochemical influence. Science of The Total Environment. 2008; 406: 337-343.

Tukivene A. Responses of fish to polycyclyc aromatic. Annales Zoologici Fennici. 1995; 32: 295-309.

Valavanidis A, Vlachogianni T, Triantafillaki S, Dassenakis M, Androutsos F, Scoullos M. Polycyclic aromatic hydrocarbons in surface seawater and in indigenous mussels (Mytilus galloprovincialis) from coastal areas of the Saronikos Gulf (Greece). Estuarine Coastal and Shelf Science. 2008; 79: 733-739.

Vanzella TP, Martinez CBR, Cólus IMS. Genotoxic and mutagenic effects of diesel oil water soluble fraction on a neotropical fish species. Mutation Research/Genetic Toxicology and Environmental Mutagenesis. 2007; 631: 36-43.

Wahidulla S, Rajamanickam Y. Detection of DNA damage in fish Oreochromis mossambicus induced by co-exposure to phenanthrene and nitrite by ESI-MS/MS. Environmental Science and Pollution Research. 2010; 17: 441-452.

Whyte JJ, Jung RE, Schmitt CJ, Tillitt DE. Ethoxyresorufin-O-deethylase (EROD) Activity in Fish as a Biomarker of Chemical Exposure. Critical Reviews in Toxicology. 2000; 30: 347-570.

Yin $\mathrm{Y}$, Jia H, Sun Y, Yu H, Wang X, Wu J, Xue Y. Bioaccumulation and ROS generation in liver of Carassius auratus, exposed to phenanthrene. Comparative Biochemistry and Physiology Part C: Toxicology \& Pharmacology. 2007; 145: 288-293.

Yuan SY, Chang JS, Yen JH, Chang B.-V. Biodegradation of phenanthrene in river sediment. Chemosphere. 2001; 43: 273-278. 